Revue internationale P.M.E.

Économie et gestion de la petite et moyenne entreprise

\title{
Portage commercial et PMI exportatrice : nature et déterminants de la création de valeur
}

\section{Pascale Bueno Merino}

Volume 16, numéro 2, 2003

URI : https://id.erudit.org/iderudit/1008440ar

DOI : https://doi.org/10.7202/1008440ar

Aller au sommaire du numéro

Éditeur(s)

Presses de l’Université du Québec

ISSN

0776-5436 (imprimé)

1918-9699 (numérique)

Découvrir la revue

Citer cet article

Bueno Merino, P. (2003). Portage commercial et PMI exportatrice : nature et déterminants de la création de valeur. Revue internationale P.M.E., 16(2), 83-104. https://doi.org/10.7202/1008440ar
Résumé de l'article

L'objet de cet article est de définir les intérêts stratégiques de l'accord de portage commercial pour la PMI exportatrice ainsi que les facteurs de succès de la coopération. Dans un premier temps, nous tenterons d'élaborer une définition du portage commercial à partir des travaux réalisés en matière de coopération symbiotique et des informations obtenues lors de nos entretiens exploratoires. Dans un deuxième temps, nous analyserons en termes de flexibilité les avantages stratégiques d'un tel partenariat pour la PMI exportatrice. Enfin, nous nous attacherons aux déterminants de la valeur partenariale. 


\section{Portage commercial et PMl exportatrice: nature et déterminants de la création de valeur}

Pascale BUENO MERINO1

Université de Caen

\section{MOTS CLÉS}

\section{Portage commercial - Portage commercial (piggyback) \\ PMI exportatrice - Coopération horizontale - Contrat d'agence Création de valeur - Flexibilité - Proximité relationnelle Innovation}

\section{RÉSUMÉ}

L'objet de cet article est de définir les intérêts stratégiques de l'accord de portage commercial pour la PMI exportatrice ainsi que les facteurs de succès de la coopération. Dans un premier temps, nous tenterons d'élaborer une définition du portage commercial à partir des travaux réalisés en matière de coopération symbiotique et des informations obtenues lors de nos entretiens exploratoires. Dans un deuxième temps, nous analyserons en termes de flexibilité les avantages stratégiques d'un tel partenariat pour la PMI exportatrice. Enfin, nous nous attacherons aux déterminants de la valeur partenariale.

\section{L'AUTEURE}

Pascale Bueno Merino est attachée temporaire d'enseignement et de recherche à l'Université d'Angers (France) et membre du CIME (Caen Innovation Marché Entreprise - IAE de Caen France). Ses recherches portent principalement sur l'internationalisation des PME et la coopération interentreprises. Elle prépare actuellement une thèse consacrée aux accords de portage commercial. Courriel : <pascale.bueno-merino@wanadoo.fr>.

1. Je tiens à remercier les lecteurs anonymes de la Revue pour la richesse de leurs commentaires. Je souhaiterais également exprimer toute ma gratitude aux praticiens interrogés dans le cadre de cette recherche. 


\begin{abstract}
The aim of this article is to identify the strategic interests of piggyback agreement for the exporting SMI and the success factors of cooperation. First of all, we try to develop a definition of piggyback from works realized as regards symbiotic cooperation and piece of information we obtained during our exploratory interviews. Then, we analyse through the concept of flexibility the strategic advantages of such a cooperation for the exporting SMI. Lastly, we study the determinants of relationship value.
\end{abstract}

\title{
RESUMEN
}

El fin de este articulo es de identificar los intereses estratégicos del acuerdo de piggyback por la pequeña y mediana industria exportadora y los factores de éxito de la cooperación. En primer lugar, tentaremos de elaborar una definición del piggyback a partir de los trabajos realizados en materia de cooperación simbiotica y de las informaciónes obtenidas durante nuestras entrevistas exploratorias. En secundo lugar, analizaremos a través del concepto de flexibilidad las ventajas estratégicas de tal cooperación por la pequeña y mediana industria exportadora. Por último, estudiaremos los determinantes del valor cooperativo.

\section{ZUSAMMENFASSUNG}

Der Gegenstand dieses Artikels besteht darin, die strategischen Interessen des Handelslieferungsabkommens für den Export kleiner und mittlerer Industrieunternehmen sowie die Faktoren der Erfolge der Zusammenarbeit zu identifizieren. In einer ersten Phase werden wir auf der Grundlage der realisierten Arbeiten versuchen, eine Definition der Handelslieferungen auszuarbeiten, die eine partnerschaftiche Zusammenarbeit kennzeichnen. In einer zweiten Stufe werden wir die strategischen Vorteile einer solchen Partnerschaft für die exportierenden kleinen und mittleren Industrieunternehmen analysieren - alles aus dem Blickwinkel der Flexibilität. Schließlich werden wir uns um die Bestimmungsfaktoren des partnerschaftlichen Wertes bemühen.

\section{Introduction}

En raison d'une insuffisance structurelle de moyens, la PME peut éprouver des difficultés dans la mise en œuvre d'une stratégie d'internationalisation. En effet, l'activité d'exportation suppose le développement de compétences spécifiques notamment en matière de prospection et de négociation. Dans cette perspective, le recours à la coopération interentreprises permet de lever certains obstacles liés à l'existence de barrières à l'entrée et peut constituer une solution créatrice de valeur pour l'entreprise de taille petite ou moyenne. Les coopérations horizontales, conclues entre entreprises concurrentes ou complémentaires, connaissent un grand succès; en témoignent les pratiques de distribution croisée entre firmes de taille semblable et, plus récemment, la multiplication des accords de portage commercial 
par quelques grands groupes industriels français ${ }^{2}$. Il s'agit de « coopérations symbiotiques », autrement dit, de collaborations concernant les maillons aval de la chaîne de valeur : marketing, ventes et services aux clients. Les travaux réalisés en matière de marketing symbiotique et entrepris par Lee Adler en 1966 attestent également de l'intérêt accru des chercheurs pour cette catégorie de coopération interentreprises ${ }^{3}$.

Le portage commercial, connu aussi sous l'anglicisme piggyback, peut être caractérisé de la façon suivante : il correspond à l'utilisation temporaire par une PMI innovante du système de distribution international d'une grande entreprise industrielle moyennant le paiement d'une commission. L'accord de portage commercial est matérialisé par la signature d'un contrat d'agent commercial conclu entre la PMI et une filiale commerciale du groupe industriel implantée à l'étranger. L'étude de cette coopération horizontale se justifie par la création de valeur qu'elle autorise pour la PMI. En effet, la vente d'une solution, par l'intégration du produit de la PMI dans l'offre industrielle d'une grande entreprise, permet une satisfaction plus étendue du client étranger et facilite par conséquent l'obtention d'un avantage concurrentiel. Outre l'accroissement de valeur perçue par le client, il faut insister sur la flexibilité engendrée par l'accord et procurant à l'entreprise de taille modeste des gains d'efficience et d'efficacité par l'optimisation de sa chaîne de valeur. Le succès de cette coopération implique toutefois une stabilité de la relation dans le temps afin de maximiser la valeur partenariale et de conforter la croissance du chiffre d'affaires de la PMI exportatrice sur le ou les marchés concernés par l'accord.

L'objet de cet article est de présenter les premiers résultats d'une étude empirique consacrée au portage commercial. Ces premiers résultats ont été obtenus grâce à la réalisation d'entretiens exploratoires menés auprès de deux PMI exportatrices et de deux groupes industriels porteurs ${ }^{4}$. Complétée par une revue de la littérature sur les liens entre producteur et intermédiaire à l'étranger, l'analyse croisée des données nous permet d'esquisser une réponse à l'interrogation suivante : Quels facteurs vont influencer la durabilité et l'efficacité du partenariat entre une PMI exportatrice et une filiale locale? De cette stabilité dépendra en effet la maximisation du gain relationnel, gain analysé principalement en termes de flexibilité pour la petite ou moyenne entreprise. La fragilité de la coopération horizontale, expliquée par l'absence de lien input-output entre les partenaires, justifie notre interrogation. Dans le cas de l'accord de portage commercial, il importe de savoir comment la PMI exportatrice parvient à entretenir l'intérêt de la filiale locale pour son produit. Autrement formulé, quels sont les déterminants de la motivation de

2. Essentiellement Rhodia (300 accords) et Pechiney (150 accords).

3. Voir particulièrement les travaux de Varadarajan et Rajaratnam (1986) et plus récemment ceux de Brock Smith (1997) et de Milliot (1998).

4. Ces entretiens ont été réalisés en avril et mai 2002.

Revue internationale P.M.E., vol. 16, $\mathrm{n}^{\circ}$ 2, 2003

(C) 2003 - Presses de l'Université du Québec 
l'agent local de représentation? Cette motivation ainsi que la création de valeur pour la PMI exportatrice seront fonction, nous le verrons, de la mise en œuvre d'une stratégie volontariste du partenaire de taille modeste, stratégie articulée autour de deux comportements fondamentaux : proximité relationnelle et innovation. Nous nous intéresserons pour finir aux motifs de rupture de l'accord et mettrons en avant les limites actuelles de notre recherche.

\section{Le portage commercial : un partenariat symbiotique}

Avant d'envisager la création de valeur suscitée par l'accord de portage commercial et les facteurs de succès de la relation entre une PMI exportatrice et une filiale locale, nous tenterons d'élaborer une définition du portage commercial à partir des travaux réalisés sur la coopération symbiotique et des informations obtenues lors de nos entretiens exploratoires. Afin d'appréhender les spécificités de l'accord de portage commercial, deux PMI exportatrices dont nous préserverons l'anonymat ont été interrogées. L'une ${ }^{5}$ exerce son activité dans l'industrie chimique et a été portée par Rhodia ${ }^{6}$ durant dix années sur les marchés japonais et chinois ; l'autre ${ }^{7}$ est actuellement portée par Pechiney en Allemagne et intervient dans l'industrie métallurgique. Nous avons également sollicité les deux groupes porteurs concernés par les accords précédemment décrits: Rhodia et Pechiney ${ }^{8}$. L'analyse croisée des données issues des quatre entretiens exploratoires a permis de faire ressortir la nature de la symbiose et d'insister sur le contenu du contrat. Nous essaierons par ailleurs de caractériser le degré de dépendance de la PMI exportatrice à l'égard de son représentant commercial.

\subsection{Entre marché et hiérarchie}

Cet accord commercial de représentation de la PMI à l'étranger revêt un caractère stratégique pour les deux partenaires et peut donc être assimilé à un véritable «partenariat symbiotique » (Koenig, 1996). Concernant le «porté »: il répond à sa volonté d'expansion internationale en favorisant l'accès à des marchés de proximité (Union européenne) ou de «grande exportation» (marchés lointains : Asie, États-Unis, Moyen-Orient, Amérique latine...) et produit un effet de levier sur les ressources mobilisées par la PMI par l'externalisation d'une partie de la fonction de commercialisation. Il s'agit de minimiser les aléas liés à la pénétration d'un marché inconnu.

5. Le directeur général et commercial de la PMI était notre interlocuteur.

6. À l'époque, il s'agissait encore de l'entité Rhône-Poulenc.

7. Le directeur export de la PMI était notre interlocuteur.

8. Le directeur du département activités complémentaires de Rhodia et le directeur de Sefranex, filiale ad hoc de Pechiney World Trade, étaient nos deux autres interlocuteurs. 
Concernant le groupe «porteur » : l'accord s'inscrit dans sa stratégie commerciale et satisfait à un objectif de rentabilité des structures à l'étranger par l'accroissement du nombre de produits commercialisés par les filiales export. De façon plus précise, le groupe intègre dans son offre industrielle de nouvelles ressources technologiques détenues par une entreprise de taille plus modeste et complète sa gamme de produits, l'objectif commun étant de répondre le plus rapidement possible à la détection d'un nouveau besoin exprimé par la clientèle étrangère. Cette coopération commerciale met en lumière l'interdépendance des deux entreprises dans la réalisation de cet objectif. Par conséquent, il s'agit bien, au sens de la théorie des coûts de transaction (Williamson, 1985, 1991), d'une véritable forme intermédiaire. La récurrence des transactions entre PMI exportatrice et filiale locale et la soustraction réciproque de ressources au marché, matérialisée par un engagement mutuel de non-concurrence, attestent le caractère hybride du mode de gouvernance. L'intérêt de l'adoption de la forme intermédiaire pour la PMI réside ici dans la diminution des coûts de transaction ex ante liés à la recherche de nouveaux clients : coûts de prospection et de négociation. La forme intermédiaire va accélérer la pénétration du marché étranger en permettant à la PMI de contourner deux catégories de barrières à l'entrée : la barrière linguistique et la barrière culturelle. Notons également l'absence d'actifs spécifiques dédiés à la relation tels que l'achat d'un équipement, l'acquisition de compétences ou l'augmentation des capacités de production. Les partenaires tirent parti d'actifs déjà existants : la force de vente locale du groupe industriel en ce qui concerne la PMI exportatrice et les actifs de la PMI concourant à l'élaboration du produit complémentaire en ce qui concerne la grande entreprise. S'agissant des actifs de la PMI, nos deux groupes porteurs ont insisté sur l'importance de deux critères de sélection ${ }^{9}$ : les compétences technologiques spécifiques de la PMI et une capacité de production suffisante au moment de la conclusion de l'accord. L'absence d'actifs spécifiques dédiés à la coopération est source de flexibilité pour l'entreprise de taille modeste. En effet, la rupture de l'accord n'occasionnera pas de coûts irrécupérables substantiels et les coûts irrécupérables se limiteront à l'investissement relationnel ${ }^{10}$ de la PMI exportatrice au cours de la relation d'intermédiation.

9. Rhodia et Pechiney ont relativisé l'importance du ratio export. À titre d'exemple, la PMI portée par Pechiney ne réalisait que $15 \%$ de ses ventes à l'étranger au moment de la conclusion du contrat d'agence.

10. L'investissement relationnel de la PMI exportatrice se rapporte aux coûts engendrés par la fréquence des contacts ou «proximité relationnelle» entre le mandant et son mandataire. Voir infra.

Revue internationale P.M.E., vol. 16, $\mathrm{n}^{\circ}$ 2, 2003

(C) 2003 - Presses de l'Université du Québec 


\subsection{Des avantages réciproques}

En biologie, la symbiose correspond à une association durable et réciproquement profitable entre deux organismes vivants. Par conséquent et métaphoriquement ${ }^{11}$, toute collaboration interentreprises peut être qualifiée de symbiotique à partir du moment où elle s'inscrit dans la durée et qu'elle crée une valeur partenariale. Cette valeur partenariale, caractérisée par l'existence d'avantages réciproques pour les symbiotes, est à l'origine du comportement coopératif. A contrario, la diminution de la valeur partenariale pour l'un des coopérants risque de compromettre la durabilité et l'efficacité de l'association. Avant d'envisager les facteurs de succès de l'accord de portage commercial, il est important de mettre en exergue les avantages de la coopération pour chacun des partenaires. Nous raisonnerons à partir des deux catégories d'avantages stratégiques mis en avant par Porter (1986): l'avantage coût et l'avantage différenciation. S'agissant de la PMI exportatrice, l'avantage coût doit être analysé comparativement à la croissance interne ou bien au recours à un agent à carte unique. Ces deux solutions apparaissent beaucoup plus onéreuses pour une PMI nouvellement exportatrice sur un marché extérieur. L'avantage coût s'explique notamment par les économies d'échelle réalisées par la filiale export du groupe porteur et répercutées sur le coût de distribution des produits de la PMI. La filiale locale distribue en effet simultanément les produits de plusieurs entreprises indépendantes; elle n'est pas l'agent exclusif d'une seule petite ou moyenne entreprise. L'investissement financier de la PMI exportatrice se limite dans le cas du portage commercial au paiement d'une commission sur les ventes réalisées à l'étranger, commission estimée raisonnable par nos deux PMI interrogées. Quant à l'avantage différenciation, il résulte de l'insertion du produit de la PMI dans une offre globale. L'importance du capital notoriété de la grande entreprise favorise l'accès au segment de marché étranger en fournissant à la PMI exportatrice la crédibilité nécessaire au démarrage d'une activité sur le marché concerné par l'accord. La valeur perçue du produit de la PMI est améliorée grâce à l'actif réputation du groupe industriel. La PMI portée dispose alors d'un avantage concurrentiel sur d'autres entreprises de taille semblable, l'accès au marché étranger constituant un facteur clé de succès pour la commercialisation d'un produit de niche. S'agissant maintenant du groupe industriel porteur et de sa filiale locale, l'avantage coût procède, nous l'avons évoqué précédemment, de l'optimisation du système de distribution international. La commercialisation de produits intermédiaires autres que ceux fabriqués par le groupe porteur entraîne la réalisation d'économies d'échelle par répartition des coûts fixes de distribution sur un plus grand nombre de produits vendus à l'étranger. L'avantage différenciation, quant à

11. Lee Adler, dans ses travaux précurseurs consacrés au «marketing symbiotique », utilisait déjà la métaphore biologique en citant l'exemple du héron et du rhinocéros (1966).

Revue internationale P.M.E., vol. 16, nº 2, 2003

(C) 2003 - Presses de l'Université du Québec 
lui, réside dans l'amélioration des capacités de négociation commerciale de la grande entreprise. En proposant une solution à son client industriel étranger, c'est-àdire une offre complète de produits intermédiaires par l'intégration de produits de niche innovants, le groupe porteur améliore également la valeur perçue de son offre, «ce qui tend à supprimer toute bataille sur les prix », nous a déclaré le directeur du département activités complémentaires du groupe Rhodia.

\subsection{Une coopération horizontale de long terme}

Attachons-nous tout d'abord à la nature des rapports entre PMI exportatrice et filiale locale. La relation est horizontale en raison de l'absence de lien input-output entre les partenaires. La filiale locale est un intermédiaire entre la PMI exportatrice et son client industriel étranger. Les deux partenaires n'entretiennent aucun rapport de filière, contrairement aux relations entre sous-traitant et grand donneur d'ordres où le produit semi-fini de l'un est intégré à la production de l'autre. Il s'agit d'une relation d'agence et non d'une relation verticale caractérisée par un transfert de droits de propriété du fournisseur au client. Par ailleurs, l'accord de portage commercial est conclu entre entreprises non concurrentes. Les produits destinés à un même segment de clientèle doivent être complémentaires pendant la relation de partenariat afin d'éviter tout risque de cannibalisation de l'offre.

Considérons maintenant la dimension temporelle de l'accord de portage commercial. Nos différents interlocuteurs ont souligné le caractère durable de la coopération. «Cinq, dix, vingt ans! » Les caractéristiques temporelles des deux accords étudiés sont les suivantes : 10 ans en ce qui concerne l'entreprise portée par Rhodia ${ }^{12}$ et six ans en ce qui concerne l'entreprise portée par Pechiney ${ }^{13}$. Cependant, la durée de la coopération n'est pas source de rigidité. En effet, est prévue au contrat une possibilité de rupture annuelle à l'initiative de l'un ou l'autre des partenaires. La durabilité de l'accord est favorisée, quant à elle, par l'existence d'une clause de reconduction tacite. Il s'agit donc d'un accord très flexible permettant, nous le verrons ultérieurement, la levée de différentes options organisationnelles. Toutefois, la contrepartie de cette flexibilité réside dans la fragilité du partenariat. L'absence de dépendance économique entre les partenaires et d'actifs spécifiques dédiés à la relation contribue à cette fragilité. Brock Smith (1997) insiste sur la fragilité des coopérations horizontales liant exportateurs et agents commerciaux. La coopération horizontale, en raison de l'absence de lien inputoutput, « tend à être volontaire, plus interpersonnelle et par conséquent plus fragile ».

12 Cette relation est achevée; elle s'est déroulée de 1985 à 1995.

13. Cette relation est en cours ; elle a été conclue pour trois ans en 1997 et a été renouvelée pour trois années supplémentaires, la PMI envisageant après l'accord de recourir aux prestations d'un agent à carte unique.

Revue internationale P.M.E., vol. 16, nº 2, 2003

(C) 2003 - Presses de l'Université du Québec

Édifice Le Delta I, 2875, boul. Laurier, bureau 450, Sainte-Foy, Québec G1V 2M2 - Tél. : (418) 657-4399 - www.puq.uquebec.ca

Tiré de : Revue internationale P.M.E., vol. 16, n² 2, sous la direction de Pierre-André Julien. 
Dans le cas d'un accord de portage commercial, la PMI exportatrice doit également gérer cette fragilité liée à l'existence d'autres partenaires symbiotiques. En effet, pour rentabiliser leur système de distribution et compléter leur offre industrielle par des produits toujours plus innovants, les groupes porteurs ont constitué des portefeuilles de compétences complémentaires. Les filiales implantées à l'étranger représentent un grand nombre d'entreprises indépendantes; la PMI exportatrice n'est donc qu'un partenaire parmi d'autres. Par ailleurs, et contrairement aux partenariats verticaux qui reposent sur une interdépendance économique, la stabilité de l'accord de portage commercial dépend exclusivement d'une donnée immatérielle qui est la motivation de l'agent local de représentation. La mise en œuvre d'une stratégie volontariste d'exportation de la PMI, révélée par différents comportements, influera sur la durabilité du partenariat et, par conséquent, sur le succès de l'accord analysé ci-dessous en termes de création de valeur pour la PMI exportatrice. Cette stratégie volontariste consistera à entretenir la motivation de la filiale locale et à se démarquer des autres mandants.

\section{Contenu de l'accord et rapports de force}

L'étude d'un contrat type fourni par l'un de nos quatre interlocuteurs a favorisé la mise en exergue des clauses contractuelles de l'accord de portage commercial. Il s'agit d'un contrat d'agent commercial conclu entre la PMI exportatrice et une filiale locale de la grande entreprise industrielle ${ }^{14}$. Par ailleurs, la confrontation des points de vue de la PMI et des grandes entreprises a permis d'appréhender la nature des rapports de force et de caractériser le degré de dépendance de la PMI à l'égard de son mandataire.

\subsection{Un contrat d'agence}

En qualité d'agent commercial, la filiale locale s'engage à prospecter et à négocier sur le marché étranger concerné par l'accord au nom et pour le compte de la PMI exportatrice. Le contrat d'agent se distingue ainsi du contrat de distribution selon lequel l'intermédiaire étranger achète pour son propre compte et revend avec profit les produits (Pasco, 2001). En d'autres termes, le distributeur est un «intermédiaire qui acquiert en plus la propriété des biens » (Boronad, Massabie-François et al., 2002). A contrario, dans le cas de l'accord de portage commercial, la filiale locale est simplement mandataire de la PMI exportatrice. L'accord de portage commercial n'opère aucun transfert de droits de propriété entre le mandant,

14. Notons que la PMI exportatrice a la faculté de contracter avec plusieurs filiales locales de la grande entreprise. Ainsi, nos deux PMI interrogées ont conclu chacune deux contrats d'agent commercial. La PME portée par Rhodia a été représentée au Japon et en Chine et la PME portée par Pechiney, en Allemagne et en Russie.

Revue internationale P.M.E., vol. 16, $n^{\circ}$ 2, 2003 
c'est-à-dire la PMI exportatrice, et son mandataire, la filiale du groupe porteur. L'agent commercial est rémunéré par le prélèvement d'une commission sur le chiffre d'affaires réalisé par la PMI exportatrice. Rappelons qu'en l'espèce la filiale locale est un «agent multicarte ». Elle dispose d'un portefeuille de mandants et ne consacre pas toute son activité à la représentation d'une seule entreprise, par opposition à l'«agent à carte unique» qu'on appelle également «agent exclusif». L'exclusivité concerne uniquement le territoire sur lequel vont être commercialisés les produits de la PMI exportatrice. La clause d'exclusivité territoriale réciproque correspond aux engagements suivants : la filiale locale s'engage à ne pas commercialiser des produits concurrents de ceux de la PMI exportatrice sur le territoire géographique mentionné au contrat et la PMI à ne pas recourir à d'autres moyens de distribution sur le même territoire. Cette clause de non-concurrence réciproque permet d' « assurer la continuité des relations » (Ménard, 1997) au cours de la représentation commerciale et facilite pour chaque coopérant l'obtention d'un avantage concurrentiel. En ce qui concerne la PMI, l'utilisation exclusive d'une partie de l'actif de distribution d'une grande entreprise favorise par rapport à d'autres concurrents de taille semblable l'accès à un nouveau segment de marché. L'accès aux marchés étrangers constitue généralement un facteur clé de succès pour les entreprises fabriquant des produits de niche. En effet et selon Saporta (1997), le «développement international est devenu pour beaucoup de PME le prolongement naturel de leur choix d'une spécialisation étroite ». Les PME bénéficiant d'un accès privilégié aux marchés étrangers disposent par conséquent d'un avantage sur leurs concurrents de taille semblable. En ce qui concerne la grande entreprise, la commercialisation exclusive, par sa filiale, de produits innovants complémentaires permet d'élargir son offre industrielle et d'augmenter la valeur commerciale perçue. Le contrat est renouvelable par tacite reconduction tous les ans et peut être rompu à l'initiative de l'un des partenaires moyennant le respect d'un préavis ${ }^{15}$; il est à durée indéterminée.

Le concept de chaîne de valeur peut apporter un éclairage utile dans la compréhension des rôles respectifs des co-contractants. Sont externalisées par la PMI exportatrice les activités de prospection et de négociation. Par le recours à des compétences externes, la PMI essaie d'optimiser le maillon «marketing et ventes » de sa chaîne de valeur. En effet, l'accès aux compétences de la filiale locale permet de réduire les coûts de transaction ex ante, c'est-à-dire les coûts liés à la recherche de nouveaux clients sur le territoire étranger et les coûts liés à la négociation du contrat. De cette façon, la PMI exportatrice économise des frais de déplacement, de traduction, d'étude du système juridique étranger, de rédaction des contrats, etc.

15. Le préavis est de six mois avant l'expiration de la période en cours. Chaque année, la filiale locale doit indiquer ses prévisions de vente pour l'année suivante. 
La compétence culturelle et linguistique de la filiale locale procure à la PMI exportatrice des gains d'efficience. À cette double compétence s'ajoute une très bonne connaissance des intervenants du secteur (clients industriels, concurrence locale...). Lu et Beamish (2001) ont à cet égard insisté sur la complémentarité entre partenaire local et PME exportatrice. Les obligations de la filiale locale concernent donc des opérations précontractuelles : prospection, négociation des contrats de vente en fonction des directives transmises par la PMI et prise de commande. À cet égard, le contrat prévoit des visites régulières de la clientèle ainsi que la fourniture de rapports sur l'évolution du marché et de la concurrence. Néanmoins, la PMI n'externalise pas l'intégralité des activités caractéristiques du maillon distribution. Selon les termes du contrat, elle doit assumer les opérations postcontractuelles : elle reste maître de l'exécution des contrats de vente conclus avec des clients étrangers. Après communication par la filiale locale d'informations relatives aux acheteurs étrangers (nom du client, coordonnées...), la PMI exportatrice prend en charge les activités suivantes : la facturation, la livraison des produits et l'assistance technique du client étranger. Par conséquent, elle entre en contact direct avec son client étranger. En assurant l'administration des ventes, elle dispose d'un fort contrôle sur son segment de marché étranger. Notons par ailleurs qu'elle supporte également les risques commerciaux liés à l'insolvabilité des clients étrangers. En conséquence, la satisfaction et la fidélisation du client industriel étranger vont dépendre de la bonne exécution du contrat de vente, autrement dit, des efforts fournis par la PME. La satisfaction de la clientèle étrangère, composée en majorité de clients du groupe porteur, influera directement sur la motivation de la filiale locale.

L'importance du contrat, en tant que régulateur des transactions, doit cependant être relativisée. Nos quatre interlocuteurs ont insisté sur le rôle prédominant de la confiance et sur l'existence d'un «contrat moral» entre les partenaires. En effet, la nature des relations interpersonnelles, analysée ci-après en termes de «proximité relationnelle», va influencer fortement le succès de la coopération. L'exemple de la PMI exportatrice portée par Rhodia est révélateur à cet égard puisque la coopération entre la PMI et le groupe porteur a duré près de dix ans, et ce en l'absence de contrat écrit. La confiance réciproque devient alors le mécanisme de gouvernance des «contributions non contractualisables» (Brousseau, 2000), c'est-à-dire des efforts de chaque partenaire consentis dans la relation de coopération et permettant l'optimisation des résultats. En raison de l'absence de lien input-output et d'actifs spécifiques dédiés à la relation ${ }^{16}$, ${ }^{1}$ investissement relationnel, favorisé par l'étroitesse des contacts interpersonnels, devient le facteur

16. En dehors des coûts engendrés par la fréquence des contacts: coups de téléphone, télécopies, déplacements à l'étranger... 
clé de succès de la coopération. En outre, l'absence de dépendance technologique ${ }^{17}$ entre les partenaires donne à la dimension interpersonnelle une importance particulière. La création de valeur pour la PMI exportatrice dépendra de son comportement volontariste au cours de l'accord de coopération. La confiance réciproque est d'autant plus importante que les co-contractants sont éloignés géographiquement. L'implication relationnelle de la PMI dans l'accord de portage commercial permettra par ailleurs de contrôler les agissements de la filiale locale et d'échapper à d'éventuels comportements opportunistes facilités par l'éloignement géographique (visites irrégulières de la clientèle étrangère par exemple). Nous verrons ultérieurement quels facteurs entretiennent la confiance réciproque des partenaires.

\subsection{Une dépendance réciproque}

Appréhendons maintenant la nature des rapports de force. Le critère de dépendance, c'est-à-dire d'aliénation de l'autonomie, est susceptible d'étoffer notre travail de théorisation de l'accord de portage commercial. À cet égard, Milliot (1998) met en exergue deux catégories de dépendance caractéristiques des coopérations symbiotiques : les « dépendances marquées unilatéralement » et les « dépendances marquées multilatéralement». Les dépendances marquées unilatéralement correspondent à des «relations caractérisées par une relative domination de l'un des partenaires ». Quant aux dépendances marquées multilatéralement, elles se rapportent à des «relations marquées par le relatif équilibre des forces en présence». S'agissant des dépendances marquées unilatéralement, l'auteur présente cinq formes de collaboration ${ }^{18}$ dont le piggyback. En effet, le portage commercial peut a priori sembler risqué pour le partenaire de taille modeste en raison de l'asymétrie de taille apparente. Cependant, il est important de rappeler que la PMI exportatrice traite directement avec la filiale locale de la grande entreprise; la filiale locale est juridiquement et dans les faits le véritable partenaire de la PMI. Or la filiale locale est généralement une entreprise de taille modeste, ce qui exclut en grande partie l'asymétrie culturelle des coopérations conclues entre PME et grandes entreprises. C'est l'exemple très souvent cité des partenariats d'impartition liant de grands donneurs d'ordres à des sous-traitants de petite taille. Le rôle de la société mère ${ }^{19}$ dans la conclusion d'un accord de portage commercial se limite aux activités suivantes : centraliser les appels d'offre des grands donneurs d'ordres étrangers et

17. On parle également d'inséparabilité technique liée à l'intégration du produit du fournisseur dans le processus de production du client.

18. Les quatre autres formes étant: l'accord de licence simple, la franchise, la prise de participation minoritaire et unilatérale et la joint-venture avec parent dominant.

19. Le siège de Rhodia, localisé à Paris, intervient à travers son département «activités complémentaires ». Quant à Pechiney, la société mère agit par l'intermédiaire de sa filiale Sefranex, localisée également à Paris.

Revue internationale P.M.E., vol. 16, $\mathrm{n}^{\circ}$ 2, 2003

(C) 2003 - Presses de l'Université du Québec 
repérer les entreprises complémentaires, françaises ou étrangères, susceptibles d'étoffer son offre industrielle. Après identification de celles-ci, elle met en contact ces partenaires potentiels avec une ou plusieurs filiales locales. Aux yeux de nos deux PMI interrogées, le contrat est apparu équilibré ; elles n'ont ressenti aucun rapport de force au moment de la négociation avec la filiale. Il ne s'agit pas d'un contrat d'adhésion. En témoignent les éléments contractuels suivants : exclusivité réciproque, commission librement négociée, amendements possibles en ce qui concerne par exemple la confidentialité des informations délivrées à l'agent commercial...

Au plan économique, les rapports sont également équilibrés. À l'extinction de l'accord, chaque coopérant récupère l'intégralité de son pouvoir de décision sur les actifs utilisés temporairement par le partenaire. L'exploitation d'actifs déjà existants n'a pas engendré de coûts irrécupérables importants en dehors des coûts de proximité relationnelle. Cet équilibre des rapports à l'extinction de l'accord procède, nous l'avons vu, de la nature horizontale de l'accord de coopération. Les deux coopérants ne sont pas liés par un rapport de production. L'équilibre des rapports entre PMI exportatrice et filiale locale entraîne la création d'une « dépendance bilatérale » et non « unilatérale », c'est-à-dire caractérisée par la domination de l'un des partenaires.

Nous avons également interrogé nos deux PMI exportatrices sur le risque de captation de savoir-faire soulevé par Usunier (1990). En effet, d'après Usunier, le succès de la PME sur le marché étranger peut « exciter la convoitise de l'entreprise qui possède l'implantation commerciale ». Au regard des réponses formulées par nos deux PMI, il semblerait que non. Le produit de la PMI, commercialisé par la filiale de la grande entreprise, est un produit de niche. Le créneau détenu par la PMI exportatrice est jugé trop étroit par la grande entreprise pour la réalisation d'économies d'échelle. Le groupe porteur a préféré ne pas répondre à la demande en raison de l'insuffisance du volume d'affaires fourni par le créneau. Le responsable de Rhodia a précisé que par ailleurs la grande entreprise et la PME pouvaient envisager de concert des opérations amicales telles que prise de participation, création de filiales communes ou filialisation. La filialisation, d'après ce dernier, ne doit pas être assimilée automatiquement à de l'opportunisme ; ce qui importe et ce qui doit légitimer l'opération, c'est «l'existence d'intérêts réciproques »...

En conséquence, dans notre effort de définition du portage commercial, nous retiendrons les éléments suivants : la dimension symbiotique de l'accord, la nature horizontale de la coopération et l'absence de domination de l'un des partenaires au moment de la négociation, pendant l'exécution et à l'extinction de l'accord. Toutefois, concernant le risque de concurrence entre la PMI exportatrice et le groupe porteur, nous émettrons certaines réserves quant au caractère généralisable de nos propos. L'insuffisance du nombre de PMI interrogées justifie notre réticence à affirmer de façon définitive le caractère non risqué de la relation, et ce en dépit du 
constat opéré en matière de propriété industrielle. Il s'avère en effet qu'aucune de nos deux PMI interrogées n'a déposé de brevet sur le marché étranger concerné par l'accord. Est-ce un indicateur de confiance sur l'évolution de la coopération? Nous l'ignorons. De multiples raisons peuvent expliquer cette absence de protection : modestie des ressources de la PMI exportatrice ou bien difficultés d'imitabilité de son processus de production... Nos prochains entretiens nous permettront de mieux appréhender le risque de concurrence.

\section{Quelle création de valeur pour la PMI exportatrice?}

Afin de caractériser la nature de la création de valeur générée par l'accord de portage commercial, nous articulerons notre réflexion autour du concept pluridimensionnel de flexibilité. Le portage commercial contribue en effet à la flexibilité dynamique de la PMI exportatrice. Selon les cas, il peut aussi favoriser sa flexibilité stratégique.

\subsection{Efficacité et efficience du portage commercial}

L'étude de la création de valeur est centrée sur l'analyse du couple efficacitéefficience. L'efficacité se rapporte à la création de valeur pour le client, c'est-àdire sa satisfaction; l'efficience renvoie, quant à elle, à l'optimisation de la chaîne de valeur de l'entreprise, c'est-à-dire à l'économie de moyens entraînée par le recours à des compétences externes. La configuration optimale de la chaîne de valeur d'une entreprise résulte de la concentration de cette entreprise sur ses compétences clés et sur l'exploitation d'interconnexions entre chaînes de valeur, autrement dit, l'échange de savoir-faire. La coopération est dans cette hypothèse source de «flexibilité externe » (Tarondeau, 1999). Porter insistait déjà en 1986 sur l'importance du rapport stratégique entre produit principal et produits complémentaires dans l'accroissement de valeur perçue par le client. Le partenaire spécialisé bénéficiant d'économies d'échelle, il est de l'intérêt du producteur de lui confier les activités correspondantes afin d'améliorer le couple efficacité-efficience. En contractant un accord de portage commercial, la PMI exportatrice parvient à générer de la valeur en recourant à la compétence commerciale d'une autre entreprise ; elle obtient des gains d'efficacité en intégrant son produit à l'offre industrielle d'une grande entreprise et des gains d'efficience en économisant les coûts liés à la prospection de nouveaux clients.

En premier lieu, l'accord de portage commercial est source de «flexibilité dynamique ». La flexibilité dynamique exprime la capacité à minimiser le délai de réaction par rapport à une variable de l'environnement (Cohendet et Llerena, 1999). Elle est souvent envisagée par rapport aux activités de production, mais elle concerne également, et de plus en plus, les activités de commercialisation (Wissler,

Revue internationale P.M.E., vol. 16, nº 2, 2003 
1993). La flexibilité dynamique procurée à la PME par la conclusion d'un accord de portage commercial résulte de sa capacité à satisfaire très rapidement un nouveau besoin exprimé par une clientèle étrangère par le recours à la force de vente commerciale d'une grande entreprise. La durée du processus de pénétration du marché étranger est minimisée grâce à l'expertise de la filiale du groupe porteur, dont la connaissance du tissu économique local facilite l'activité de prospection. De plus, la réputation du groupe porteur améliore la crédibilité de la PMI exportatrice sur le marché étranger; elle permet d'obtenir plus rapidement la confiance du client industriel. L'accord de portage commercial est également source d'efficience pour la PMI, car il autorise le report des irréversibilités liées à la décision d'investissement commercial sur le marché étranger. En effet, l'externalisation d'une partie de l'activité de commercialisation permet à l'entreprise portée d'éviter de lourds investissements jugés risqués particulièrement dans un environnement inconnu. En effet, l'engagement financier de la PMI se limite au paiement d'une commission sur le chiffre d'affaires réalisé au cours de l'intermédiation. Dans cette perspective, le portage commercial favorise la rationalisation de son activité de distribution internationale et la concentration de ses ressources financières sur les activités créatrices de valeur telles que la recherche et développement.

Le report des investissements commerciaux rend possible la préservation d'options organisationnelles et améliore la réversibilité des décisions prises par la PMI en matière de commercialisation de ses produits à l'étranger. Pour Wolff (1992), l'accord interentreprises constitue « une option sur des activités nouvelles et incertaines, permettant éventuellement après-coup le repositionnement des acteurs ». «Cette flexibilité générée par le mode de gouvernance en réseau permet de repousser la décision irréversible à un instant où l'incertitude aura diminué c'est-à-dire jusqu'à l'apparition d'informations supplémentaires sur le succès de l'opération, sur le partenaire » (Joffre, 1999). Autrement dit, l'accord de portage commercial facilite l'estimation des potentialités du marché et la prise de décision concernant l'adoption d'une modalité plus « hiérarchique » de distribution. En effet, le portage commercial est une solution efficiente mais temporairement efficace lorsque la taille du marché augmente ; cette inefficacité est liée à l'importance de l'activité de représentation de la filiale locale. En cas de succès grandissant de la PMI exportatrice sur le marché concerné par l'accord, la filiale locale n'est plus en mesure de consacrer le temps nécessaire à la couverture totale du marché, et ce en raison de l'étendue de son portefeuille d'accords d'intermédiation. Par conséquent, l'augmentation de la taille du marché de la PMI exportatrice rend le portage commercial inefficace en termes de couverture du marché. Dans cette hypothèse, la valeur partenariale est menacée et la PMI se trouve dans l'obligation de recourir à d'autres modalités de distribution. Nos deux PMI interrogées ont évoqué les deux options organisationnelles suivantes : agent commercial ou distributeur de taille plus modeste, c'est-à-dire disposant d'un portefeuille d'accords de représentation commercial beaucoup moins étendu, et agent à carte unique. Ainsi, la PMI

Revue internationale P.M.E., vol. 16, nº 2, 2003

(C) 2003 - Presses de l'Université du Québec 
portée par Rhodia s'est adressée, après l'accord de portage, à trois autres intermédiaires de taille réduite pour commercialiser ses produits : un agent local chinois et deux petites sociétés d'importation japonaises. La PMI portée par Pechiney nous a précisé qu'à l'extinction de l'accord elle recourrait très probablement aux prestations d'un agent exclusif local permettant d'assurer selon elle «le meilleur développement possible». La coopération avec Pechiney est maintenue pour des raisons financières, le recours à un agent exclusif représentant pour le moment un investissement trop important. Une autre solution de distribution a également été mise en évidence par nos deux groupes porteurs : la création par la PMI exportatrice de sa propre filiale sur le marché étranger. Cette troisième solution constitue la modalité la plus hiérarchique au sens de la théorie des coûts de transaction. Dans cette hypothèse, la PMI internalise la fonction de commercialisation dans son intégralité. Elle assume seule la prospection et la négociation des nouveaux contrats. Notons que la levée de ces différentes options organisationnelles est facilitée par le contenu du contrat. La clause de rupture annuelle favorise l'adaptation de la PMI exportatrice au contexte local. En cas d'évolution favorable de la part de marché, le portage commercial constitue une transition vers une maîtrise plus internalisée de la croissance du chiffre d'affaires de la PMI sur le marché étranger. En cas d'évolution défavorable, le portage commercial octroie des positions de repli. La PMI peut très facilement rompre son contrat et se retirer du territoire étranger. Elle peut également contracter de nouveaux accords avec d'autres filiales du groupe porteur et assurer une diffusion géographique la plus large possible de son produit innovant.

\subsection{Une flexibilité stratégique}

L'accord de portage commercial est susceptible d'améliorer la flexibilité stratégique de la PMI exportatrice. La flexibilité stratégique, par opposition à la flexibilité opérationnelle, exprime la capacité à faire face à des changements dans la nature des activités et non plus dans leur niveau (Reix, 1979). L'accord de portage commercial est générateur de flexibilité stratégique parce qu'il peut être à l'origine de la création de nouveaux produits. La mobilité des ressources de la PMI exportatrice résulte ici d'une utilisation nouvelle des biens d'équipement, autrement dit, d'une déclinaison du savoir-faire de la PMI. Elle modifie les orientations du développement stratégique de la PMI : du développement de produits à la diversification ${ }^{20}$. C'est l'expertise multisectorielle de la filiale locale qui va favoriser la flexibilité stratégique de la PMI. La filiale locale repère un nouveau couple produit-marché et fait part à la PMI exportatrice de l'existence de cette découverte. Dans cette

20. Pour Johnson, Scholes et Fréry (2002), le développement de produits consiste à proposer une offre nouvelle sur les marchés existants (nouveaux produits, marchés existants). La diversification consiste pour une entreprise à s'engager dans des domaines d'activité dans lesquels elle n'est pas encore présente (nouveaux produits, nouveaux marchés).

Revue internationale P.M.E., vol. 16, $\mathrm{n}^{\circ}$ 2, 2003

(C) 2003 - Presses de l'Université du Québec 
hypothèse, le portage commercial représente pour la PMI exportatrice non seulement un instrument de veille commerciale mais aussi un instrument de veille technologique. Il permet, d'une part, la découverte de nouveaux clients pour des produits existants et, d'autre part, la création de nouveaux produits par la découverte de nouveaux besoins. À cet égard, le responsable de l'activité portage de Pechiney a insisté sur le rôle du service export de la PMI exportatrice, qui doit assurer l'interface entre le département conception et la clientèle étrangère. Il facilite en outre l'adaptation de l'offre de la PMI aux nouveaux besoins de la clientèle en transmettant à la conception les informations communiquées par l'agent local de représentation. «Le responsable export doit se concentrer sur ce qui génère de la valeur. » L'accord de portage commercial favorise dans cette perspective l'optimisation de la chaîne de valeur de la PMI exportatrice. L'externalisation temporaire de l'activité de prospection permet à la PMI de se concentrer sur le développement technologique de son activité et d'exploiter de nouvelles opportunités liées à la découverte de nouveaux besoins. Il en résulte une modification de l'offre de la PMI par l'élargissement de sa gamme de produits ${ }^{21}$. Dans cette perspective, la PMI mène de front deux manœuvres stratégiques : l'internationalisation de son activité et une diversification de proximité. Ainsi, en desserrant la contrainte des ressources, la stratégie relationnelle permet de contourner les dilemmes classiques de la PME en termes d'options stratégiques (Puthod, 1998). Les ressources commerciales et informationnelles de la filiale locale vont faciliter la conciliation des deux manœuvres.

\section{Les facteurs de succès de la relation}

Le succès de l'accord de portage commercial pour la PMI est lié à la stabilité de la coopération dans le temps afin d'optimiser la valeur partenariale et de conforter la croissance du chiffre d'affaires de la PMI sur le ou les marchés concernés par l'accord. Cette stabilité est recherchée par la PMI en raison de l'existence d'avantages exprimés en termes de flexibilité : satisfaction rapide d'une nouvelle clientèle, report des irréversibilités et amélioration de l'offre de la PMI. Nous avons par conséquent tenté d'établir à travers les entretiens exploratoires les déterminants de cette création de valeur. Trois principaux facteurs de succès ont été mis en évidence : la proximité relationnelle entre la PMI exportatrice et la filiale locale, la satisfaction de la clientèle étrangère et la capacité d'innovation de la PMI.

21. Notons qu'à cet égard le contrat de portage commercial est également très souple. Il prévoit pour la PMI la possibilité de modifier au cours de la relation d'intermédiation sa gamme de produits. 


\subsection{La proximité relationnelle entre la PMI exportatrice et la filiale locale}

Les travaux réalisés en matière de coopération horizontale insistent sur l'aspect suivant : l'absence de lien input-output rend la coopération horizontale plus fragile et plus volontaire, comparativement à la coopération verticale entre client et fournisseur (Brock Smith, 1997). La stabilité de la coopération horizontale est donc principalement liée à la motivation de chaque partenaire qui, elle, repose sur la confiance. Cette relation entre confiance réciproque et stabilité du partenariat dans le temps est également applicable à notre objet d'étude. Toutefois, il semblerait que cette stabilité dépende essentiellement du comportement volontariste de la PMI exportatrice. En effet, eu égard à l'existence d'autres mandants, la PMI doit s'efforcer d'attirer l'attention de la filiale locale. Son comportement volontariste constitue un avantage comparatif et influe sur l'efficacité de la représentation commerciale, autrement dit, sur le temps consacré par la filiale locale à la commercialisation des produits de la PMI.

Nos quatre interlocuteurs ont souligné l'importance de la fréquence des contacts entre la PMI exportatrice et son agent de représentation. La proximité relationnelle est un facteur de succès de l'accord de coopération. Elle favorise l'instauration d'un climat de confiance réciproque (Morgan et Hunt, 1994 ; Solberg, 2001) et permet d'éprouver la motivation de chaque partenaire. La confiance réciproque procède pour partie de la bonne exécution du contrat, notamment en ce qui concerne la transparence informationnelle : rapports de visite, copie des devis émis par la filiale locale, copie des devis ou des documents de facturation émis par la PMI exportatrice lorsque des clients étrangers ont passé commande directement à la PMI sans en informer au préalable l'agent de représentation ${ }^{22} \ldots$ Toutefois, l'existence d'une confiance réciproque ne s'explique pas uniquement par le respect des obligations contractuelles. La confiance réciproque est également influencée par des comportements non prévus au contrat et justifiant d'une réelle motivation du partenaire. Ainsi, dans les faits, il semblerait que l'efficacité de la prospection, exprimée en termes de couverture du marché, dépende des capacités de suivi commercial de la PMI. Très régulièrement, elle doit contacter son agent local de représentation pour connaître l'évolution de son marché et s'assurer du caractère actif de la prospection. Elle ne doit pas se contenter d'attendre les rapports périodiques d'activité prévus au contrat. De cette façon, elle témoigne de sa motivation et attire l'attention de la filiale locale sur ses produits. Cette proximité relationnelle permet d'entretenir la motivation de la force de vente locale et contribue à la

22. Dans ce cas, le mandataire doit être averti par la PMI. Le paiement de la commission dépend de cette transparence informationnelle. À cette fin, la PMI doit fournir périodiquement un relevé de compte mentionnant les ventes réalisées sur le territoire étranger.

Revue internationale P.M.E., vol. 16, $\mathrm{n}^{\circ}$ 2, 2003

(C) 2003 - Presses de l'Université du Québec 
distinguer des autres mandants ; elle s'exerce de différentes façons : coups de téléphone, télécopies, déplacements de la PMI à l'étranger... « Il faut se manifester le plus souvent possible », a déclaré l'une de nos PMI interrogées. Rhodia et Pechiney ont particulièrement insisté sur l'importance des visites en commun de la clientèle locale. Par ailleurs, la PMI peut dans certains cas prendre part directement à la prospection. La PMI portée par Pechiney nous a rapporté qu'elle avait participé à un salon export à l'étranger en compagnie d'un représentant de la filiale locale de la grande entreprise.

\subsection{La satisfaction de la clientèle étrangère}

La bonne exécution des contrats de vente influe également sur la motivation de l'agent local de représentation. À cet égard, Pechiney met l'accent sur le «professionnalisme » de la PMI exportatrice. «La clientèle étrangère et la clientèle nationale doivent être placées sur le même pied d'égalité.» Le respect des délais de livraison et la qualité des produits vont conditionner la satisfaction et la fidélisation de la clientèle locale, composée en grande partie de clients du groupe porteur. L'assistance technique délivrée par la PMI et prévue dans le contrat d'agence vient renforcer cette satisfaction. Le professionnalisme de la PMI est un déterminant de la motivation de la force de vente locale pour deux raisons principales. En premier lieu, la réputation de la grande entreprise ne doit pas être entachée. En effet, les produits de la PMI exportatrice sont intégrés dans l'offre globale du groupe industriel. La PMI bénéficie de l'actif réputation de la grande entreprise et la mauvaise exécution du contrat de vente risque de compromettre la notoriété du groupe industriel sur le marché étranger. A contrario, la satisfaction de la clientèle incite la force de vente locale à poursuivre ses efforts de prospection pour le compte de la PMI exportatrice. Elle est un gage de durabilité de la coopération puisqu'elle influe sur la complémentarité entre produits du groupe porteur et produits de la PMI exportatrice. La création de valeur pour le client permet d'entretenir le caractère symbiotique de la coopération à travers l'existence d'avantages réciproques. En second lieu, le professionnalisme de la PMI exportatrice laisse présager des gains d'efficience pour la grande entreprise. L'augmentation du chiffre d'affaires de la PMI sur le marché étranger favorise le prélèvement de commissions et donc la rentabilisation du système de distribution de la grande entreprise. Cette perspective de rentabilité, liée à la satisfaction de la clientèle étrangère, accroît la motivation de la filiale locale dans sa recherche de nouveaux clients.

\subsection{La capacité d'innovation de la PMI exportatrice}

Le comportement innovateur de la PMI exportatrice est un autre facteur de stabilité de la coopération. Au préalable, il a constitué un critère de sélection de la PMI

exportatrice. En effet, c'est la compétence technologique spécifique de la PMI qui 
a retenu l'attention de la grande entreprise. L'amélioration continue des produits révèle la volonté de la PMI de s'adapter à l'évolution des besoins de la clientèle étrangère et permet d'entretenir la motivation de l'agent local de représentation. La capacité d'innovation de la PMI exportatrice s'exprime de différentes manières : amélioration des produits existants, création de nouveaux produits... Cette capacité d'innovation est d'autant plus importante qu'au fil du temps la PMI est exposée à un risque d'effritement progressif de la motivation de la force de vente locale à l'égard de sa gamme de produits. En effet, afin de s'adapter aux évolutions technologiques, le groupe porteur procède régulièrement à la revitalisation de son offre industrielle en intégrant de nouveaux produits complémentaires. Les filiales locales ont tendance assez naturellement à concentrer leurs efforts de prospection sur les produits complémentaires nouvellement inclus dans la gamme en raison de leur caractère novateur. L'enthousiasme de la force de vente locale pour ces nouveaux produits, justifié par l'acquisition de nouvelles compétences techniques, menace l'efficacité de la représentation commerciale des produits plus anciens. La réponse de la PMI réside alors dans sa capacité à innover et à rompre la monotonie susceptible de caractériser le travail de représentation des commerciaux locaux.

\subsection{Les causes de rupture de l'accord}

La rupture de l'accord est liée à une efficacité moindre de la coopération en termes de couverture totale du marché, autrement dit, à une diminution de la valeur partenariale. En premier lieu, cette efficacité moindre peut être liée à l'augmentation de la taille du marché de la PMI. La filiale locale, parce qu'elle commercialise à la fois les produits de la grande entreprise et ceux d'autres entreprises complémentaires, n'est plus en mesure de consacrer le temps nécessaire à la couverture totale du marché de la PMI, celle-ci devant, par conséquent, trouver une solution plus efficace de couverture du marché étranger. Elle peut recourir aux prestations d'un intermédiaire de taille plus modeste ou bien solliciter un agent à carte unique si elle dispose des ressources financières nécessaires. De cette manière, elle procède à une maîtrise plus «internalisée» de la croissance de son chiffre d'affaires. À l'extrême, elle peut créer sa propre organisation commerciale sur le territoire étranger. Mais cette dernière solution suppose, outre des ressources financières, la détention de diverses expertises en matière de négociation, de droit, de finance... Dans ces conditions, le portage commercial est apparu comme une solution efficiente mais temporairement efficace, la PMI étant victime de son propre succès sur le marché étranger. En second lieu, la diminution des gains d'efficacité pour la PMI s'explique, nous l'avons évoqué précédemment, par l'érosion de la motivation de la force de vente locale. La propension de la filiale locale à se concentrer sur ses propres produits ou sur les produits complémentaires nouvellement intégrés dans l'offre industrielle de la grande entreprise réduit le temps consacré aux produits plus anciens et, par conséquent, l'efficacité de la représentation. Dans cette

Revue internationale P.M.E., vol. 16, $\mathrm{n}^{\circ}$ 2, 2003

(C) 2003 - Presses de l'Université du Québec 
perspective, les efforts de la filiale locale sont difficilement maîtrisables. La faiblesse du contrôle de la PMI exportatrice à cet égard est liée à la non-détention des droits de propriété du réseau de distribution. Le portage commercial est alors considéré comme un «mode de délégation souple», caractérisé par un faible contrôle du canal de distribution et par un risque financier minime, deux variables étroitement corrélées (Filser, 1989; Lemaire, 1997). «La coopération n'est pas envisagée ad vitam eternam », nous a déclaré la PMI portée par Pechiney. «Il s'agit de démarrer une activité à l'exportation... » Le portage commercial permet, d'après notre interlocuteur, de parer aux difficultés liées à la découverte d'un distributeur ou d'un agent commercial autochtone. Le comportement proactif de la PMI consiste alors à mettre en œuvre une stratégie de diversification des risques par la recherche d'une solution de distribution internationale plus efficace, susceptible d'assurer à plus long terme une représentation commerciale optimale de la PMI exportatrice.

\section{Conclusion}

En conséquence, la stabilité et l'efficacité de la coopération horizontale étudiée, mesurée par la couverture du marché, dépendent du comportement volontariste de la PMI exportatrice. Outre la bonne exécution des engagements contractuels, fréquence des contacts et comportement innovateur de la PMI influencent directement la motivation de l'agent local de représentation et permettent d'optimiser les avantages réciproques du partenariat. Toutefois, l'évolution de la valeur partenariale ne dépend pas uniquement des efforts fournis par la PMI. Au cours du temps, l'efficacité de la représentation commerciale tend à s'amoindrir notamment pour des raisons liées à la taille du portefeuille d'accords d'intermédiation de la filiale locale. La PMI doit alors envisager le recours à d'autres moyens de distribution de ses produits à l'étranger.

Pour conclure, nous insisterons sur les limites actuelles de notre recherche. Les quatre études de cas ont permis dans un premier temps:

- de mettre en exergue les spécificités de la coopération étudiée;

- de caractériser la création de valeur suscitée par le contrat d'intermédiation commerciale ;

- de dégager des hypothèses sur les facteurs de succès de la relation analysés en termes de stabilité de la coopération dans le temps et d'efficacité de la représentation commerciale.

Néanmoins, dans un deuxième temps, nous devrons nous efforcer d'apprécier le caractère généralisable de nos propos, notamment en ce qui concerne les facteurs de succès de la relation, en augmentant le nombre d'études de cas approfondies. Par ailleurs, la découverte de «cas d'échecs » permettrait peut-être de nuancer le caractère symbiotique de la coopération. 


\section{Bibliographie}

ADLER, L. (1966), «Symbiotic marketing », Harvard Business Review, novembre-décembre, p. 29-41.

Boronad, V., M. Massabie-François, M. Petitclerc, E. Poulain et L. Rota-Lonjon (2002), Commerce international - Techniques et management des opérations, Rosny, Éditions Bréal.

BROCK SMITH, J. (1997), «Selling alliances : issues and insights », Industrial Marketing Management, vol. 26, p. 149-161.

BrousseAu, E. (2000), «La gouvernance des processus de coopération », dans La coopération industrielle, Paris, Economica.

Cohendet, P. et P. Llerena (1999), «Flexibilité et modes d'organisation», Revue française de gestion, $\mathrm{n}^{\mathrm{o}} 123$, mars-avril-mai.

Desreumaux, A. (1999), «Partenariat», dans R. Le Duff (dir.), L'encyclopédie de la gestion et du management, Dalloz, p. 889-890.

FILSER, M. (1989), Canaux de distribution, Paris, Vuibert Gestion.

JOFFRE, P. (1999), «L'économie des coûts de transaction ou le marché et l'entreprise à la fin du $20^{\text {ème }}$ siècle», dans De nouvelles théories pour gérer l'entreprise du $21^{\text {ème }}$ siècle, coordonné par G. Koenig, Paris, Economica, p. 143-170.

Johnson, G., K. Scholes et F. Frery (2002), Stratégique, Paris, Pearson Education.

KoENIG, G. (1996), Management stratégique, Paris, Nathan.

LEMAIRE, J.-P. (1997), Stratégies d'internationalisation, Paris, Dunod.

LU, J.W. et P.W. BEAMISH (2001), «The internationalization and performance of SMEs », Strategic Management Journal, vol. 22, p. 565-586.

MÉNARD, C. (1997), «Le pilotage des formes organisationnelles hybrides », Revue économique, vol. 48, $\mathrm{n}^{\mathrm{o}} 3$, p. 741-750.

MiLliot, E. (1998), Le marketing symbiotique - la coopération au service des organisations, Paris, L'Harmattan.

MorGAn, R.M et S.D. HunT (1994), «The commitment-trust theory of relationship marketing », Journal of Marketing, vol. 58, p. 20-38.

PASCO, C. (2001), Commerce international, Paris, Dunod.

PORTER, M. (1986), L'avantage concurrentiel de l'entreprise, Paris, InterÉditions.

Puthod, D. (1998), «L'alliance, une option stratégique permettant de contourner les dilemmes classiques de la PME», dans PME, de nouvelles approches, sous la coordination d'O. Torrès, Paris, Economica.

REIX, R. (1979), La flexibilité de l'entreprise, Paris, Cujas.

SAPORTA, B. (1997), «Stratégies des petites et moyennes entreprises », dans P. Joffre et Y. Simon (dir.), L'encyclopédie de gestion, $2^{\mathrm{e}}$ édition, tome 3, Paris, Economica, p. 3105-3128.

Revue internationale P.M.E., vol. 16, $\mathrm{n}^{\circ}$ 2, 2003

(C) 2003 - Presses de l'Université du Québec 
SolBERG, C.-A. (2001), «Modes of exporter governance of sales subsidiaries and distributors in international markets : a literature review and model », Cahier de recherche LAREMA, février 2001, Groupe ESC Bordeaux.

TARONDEAU, J.-C. (1999), La flexibilité dans les entreprises, Paris, Presses universitaires de France, coll. «Que sais-je?», nº 3477.

USUNIER, J.-C. (1990), Environnement international et gestion de l'exportation, Paris, Presses universitaires de France, coll. «Gestion».

VARADARADJAN, P.R. et D. RAJARATNAM (1986), « Symbiotic marketing revisited », Journal of Marketing, vol. 50, p. 7-17.

Williamson, O. (1985), The Economic Institutions of Capitalism, New York, The Free Press.

Williamson, O. (1991), «Comparative economic organization : the analysis of discrete structural alternatives », Administrative Science Quarterly, vol. 36, nº 2, p. 269-296.

WISSLER, M. (1993), «Le portage à l'export et les stratégies relationnelles des PME», Communication au colloque «PME-PMI et développement international», Aix-en-Provence, juin.

WOLFF, S. (1992), Accords inter-entreprises et flexibilité : éléments théoriques et application au secteur des télécommunications, Thèse pour le doctorat, Université Louis-Pasteur. 